\title{
The Detailed Chemical Abundance Patterns of M31 Globular Clusters
}

\author{
Janet E. Colucci* ${ }^{\dagger}$ \\ University of California Santa Cruz \\ E-mail: jcolucci@ucolick.org
}

\section{Rebecca A. Bernstein}

University of California Santa Cruz

E-mail: rab@ucolick.org

\section{Judith Cohen}

California Institute of Technology

E-mail: jlc@astro.caltech.edu

We present detailed chemical abundances for $>20$ elements in $\sim 30$ globular clusters in M31. These results have been obtained using high resolution $(\lambda / \Delta \lambda \sim 24,000)$ spectra of their integrated light and analyzed using our original method. The globular clusters have galactocentric radii between $2.5 \mathrm{kpc}$ and $117 \mathrm{kpc}$, and therefore provide abundance patterns for different phases of galaxy formation recorded in the inner and outer halo of M31. We find that the clusters in our survey have a range in metallicity of $-2.2<[\mathrm{Fe} / \mathrm{H}]<-0.11$. The inner halo clusters cover this full range, while the outer halo globular clusters at $\mathrm{R}>20 \mathrm{kpc}$ have a small range in abundance of $[\mathrm{Fe} / \mathrm{H}]=-1.6 \pm 0.10$. We also measure abundances of alpha, $\mathrm{r}$ - and s-process elements. These results constitute the first abundance pattern constraints for old populations in M31 that are comparable to those known for the Milky Way halo.

XII International Symposium on Nuclei in the Cosmos,

August 5-12, 2012

Cairns, Australia

\footnotetext{
* Speaker.

${ }^{\dagger}$ The data presented herein were obtained at the W. M. Keck Observatory, which is operated as a scientific partnership among the California Institute of Technology, the University of California and the National Aeronautics and Space Administration. The Observatory was made possible by the generous financial support of the W. M. Keck Foundation.
} 


\section{Introduction}

Study of M31, the Milky Way's nearest massive neighbor, is interesting for many reasons. One of the most fundamental questions is whether the characteristics of the M31 spiral galaxy support the assertion that the Milky Way is a "normal" spiral galaxy. This is important because we can study the properties of the Milky Way in great detail, and studies of our own galaxy by necessity are the foundation for our understanding of how galaxies in general form and evolve. As the next closest massive galaxy, M31 is the first place to test galaxy formation theories developed from studies of the Milky Way, and in some respects is a more ideal test-case because M31 can be observed as a whole from the outside, whereas study of our own galaxy is complicated by our position within it. However, the distance to M31 means that we are unable to study its individual stars at the same level of detail that we can obtain in the Milky Way, using high resolution high signal-to-noise ratio (SNR) spectra and detailed chemical abundance analyses.

With the development of our original technique for abundance analysis of high resolution integrated light (IL) spectra of globular clusters (GCs), we can now make significant advances in chemical evolution studies of distant massive galaxies. Unresolved GCs, which are luminous and therefore observationally accessible to large distances, can be used to learn about the chemical enrichment and formation history of other galaxies, just as they were originally used to learn about the formation of the Milky Way [1]. Our technique has been developed and demonstrated on resolved GCs in the Milky Way and Large Magellanic Cloud (LMC) in a series of papers [2, 3, 4, $5,6,7]$. These works demonstrate that the IL analysis provides accurate $\mathrm{Fe}$ abundances and $[\mathrm{X} / \mathrm{Fe}]$ ratios to $\sim 0.1 \mathrm{dex}$, as well as distinguishes ages for GCs with a range in properties, including $[\mathrm{Fe} / \mathrm{H}]$ of -2 to +0 and ages from 0.05 to $12 \mathrm{Gyr}$.

With this method, we have now begun an unprecedented study of the chemical composition of the GC system of M31. Detailed abundances of $\sim 20$ elements were presented for a pilot sample of $5 \mathrm{M} 31 \mathrm{GCs}$ in [4]. As part of this ongoing project, here we extend the sample of [4] and present ages, Fe, $\alpha$-element and neutron capture abundances of an additional 22 GCs in M31.

\section{Observations}

We obtained high resolution IL spectra of the M31 GCs using the HIRES spectrograph on the Keck I telescope. The data were taken over several observing runs from 2006-2011. In all observing runs we used identical setups that utilized the D3 decker, which has a slit size of 1.7 " $\times 7.0$ " and spectral resolution of $R=24,000$, which is sufficient to resolve individual spectral lines of GCs with velocity dispersions, $\sigma_{v}>\sim 7 \mathrm{kms}^{-1}$. The wavelength coverage is approximately $3800-8300$ $\AA$. Exposure times were between $1-5$ hours for each GC. The SNR at $6000 \AA$ is approximately 60-90. Data were reduced with standard routines in the HIRedux pipeline. ${ }^{1}$

\section{Analysis}

As in all of our our previous spectroscopic analyses we first measure individual absorption line equivalent widths (EWs) using the semi-automated program GETJOB [8]. Line lists and oscillator strengths were taken from $[3,4,6]$ and references therein. In this work, we also use our IL spectral

\footnotetext{
${ }^{1}$ http://www.ucolick.org/ xavier/IDL/index.html
} 

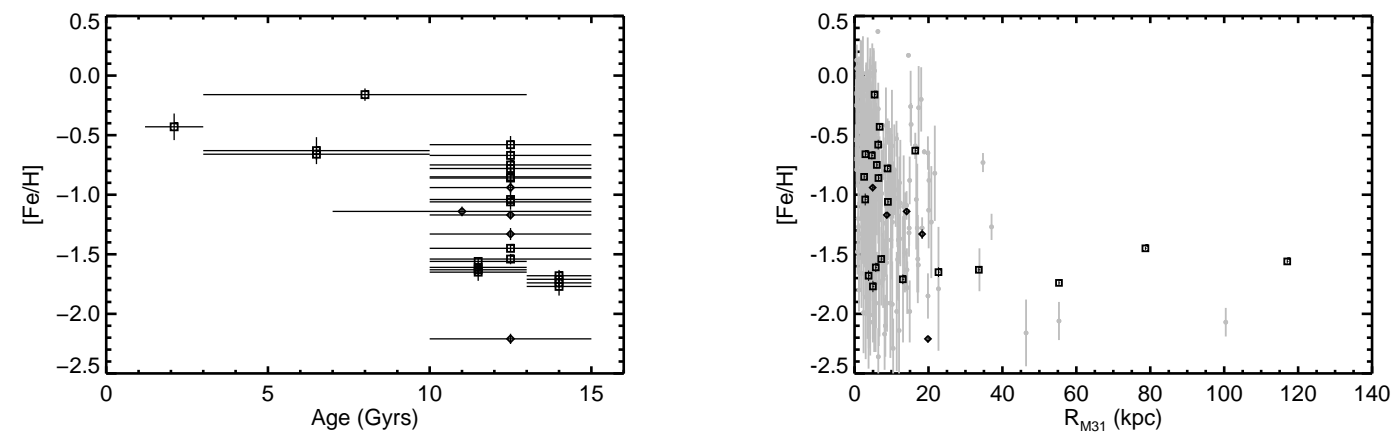

Figure 1: Left: The age-metallicity relationship. Black diamonds are from [4], and black squares are from this work. Right: The behavior of $[\mathrm{Fe} / \mathrm{H}]$ with projected galactocentric radius from $\mathrm{M} 31$, where radii are taken from [11]. Gray points are from [12], and measured using Lick system line indexes.

synthesis code [7] for the best possible accuracy in our final measurements for $\mathrm{Ca}, \mathrm{Si}, \mathrm{Eu}, \mathrm{Ba}$ and Y. Abundances for $\mathrm{Ba}$ and Eu include corrections for hyperfine splitting, as described in [7].

The IL abundance analysis is described in detail in $[3,4,5]$. In this analysis we create synthetic GC color magnitude diagrams (CMDs) using the Teramo isochrones [9] without convective overshooting, with extended asymptotic giant branch (AGB), and mass - loss parameter of $\eta=0.2$. More discussion about the testing and choice of appropriate isochrones can be found in $[3,5,6]$. Flux-weighted synthesized EWs of lines are calculated using our routine ILABUNDS [3], which utilizes spectral synthesis routines from the 2010 version of MOOG [10]. We use the ODFNEW and AODFNEW model stellar atmospheres from Kurucz. ${ }^{2}$ All abundances are calculated under the assumption of local thermodynamic equilibrium (LTE).

The age and $[\mathrm{Fe} / \mathrm{H}]$ solutions for each cluster are identified as the range in synthetic CMD ages and $[\mathrm{Fe} / \mathrm{H}]$ that produce the most self-consistent results using the 10-80 individual Fe I lines measured in each cluster. The best solutions have the smallest statistical error $\left(\sigma_{\mathrm{N}}\right)$, and minimal dependence of Fe I abundance with line excitation potential (EP), wavelength, and EW. For each cluster there is a range in CMD ages that produce similarly self-consistent solutions. For older clusters this range is typically $10-15 \mathrm{Gyr}$, and leads to an internal systematic uncertainty in $[\mathrm{Fe} / \mathrm{H}]$ of $\lesssim 0.05$ dex. For the total uncertainty in $[\mathrm{Fe} / \mathrm{H}]$ for each cluster, we add this internal systematic age uncertainty, $\sigma_{\mathrm{Age}}$, in quadrature with the statistical error in the mean abundance, $\sigma_{\mathrm{N}} / \sqrt{\mathrm{N}-1}$, where $\mathrm{N}$ is the number of lines measured for each element. The best CMD solution is used to calculate abundances for the other elements.

\section{Results and Conclusions}

In Figure 1 we show the age-metallicity relationship for the 22 M31 GCs analyzed in this work and the $5 \mathrm{GCs}$ analyzed previously [4]. Figure 1 also shows the $\mathrm{M} 31 \mathrm{GC}[\mathrm{Fe} / \mathrm{H}]$ results against M31 projected galactocentric radii from [11]. The low resolution results of [12] are also shown for comparison and to highlight the greater precision of our technique when compared to low resolution results. We find a spread in $[\mathrm{Fe} / \mathrm{H}]$ for $\mathrm{GCs}$ within $\mathrm{R}_{\mathrm{M} 31} \sim 20 \mathrm{kpc}$, and a fairly constant $[\mathrm{Fe} / \mathrm{H}]$ for $\mathrm{GCs}$ at $\mathrm{R}_{\mathrm{M} 31}>20 \mathrm{kpc}$. The mean value for the $5 \mathrm{GCs}$ in our sample that lie at $\mathrm{R}_{\mathrm{M} 31}>20$ $\mathrm{kpc}$ is $[\mathrm{Fe} / \mathrm{H}]=-1.63 \pm 0.10$. We therefore confirm the previous results of $[13,14]$, who also found a nearly constant $[\mathrm{Fe} / \mathrm{H}]$ for outer halo GCs. Like our analysis, [14] found a flat metallicity

\footnotetext{
${ }^{2}$ http://kurucz.harvard.edu/grids.html
} 

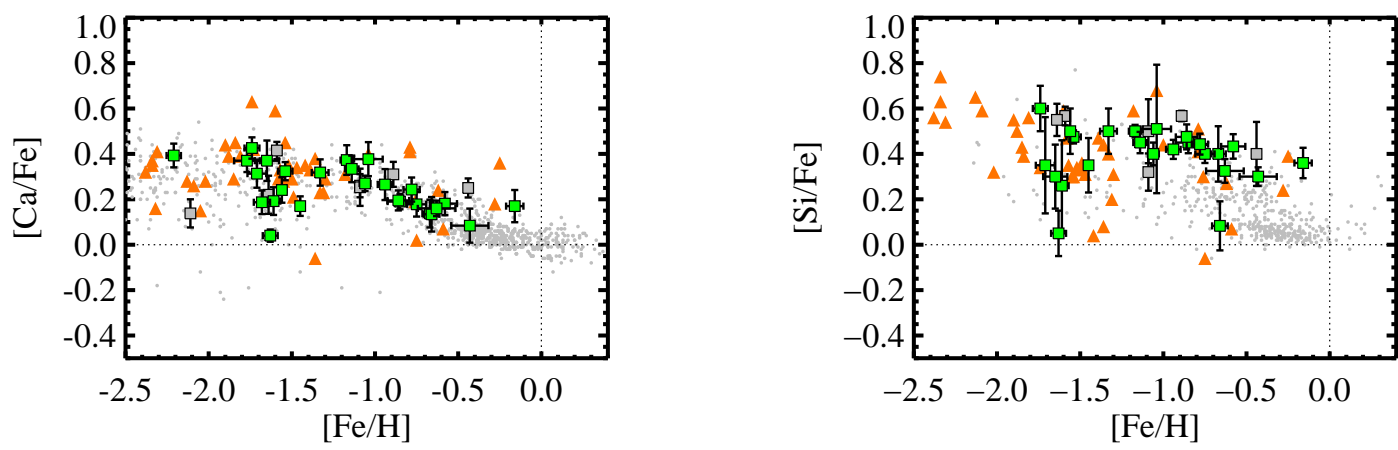

Figure 2: $\alpha$-element abundances in our sample to date. Green squares show M31 GCs, Grey squares show MW IL abundances from our training set GCs [5]. Small gray circles and orange triangles, respectively, show MW field stars [16] and MW GC stars [17].
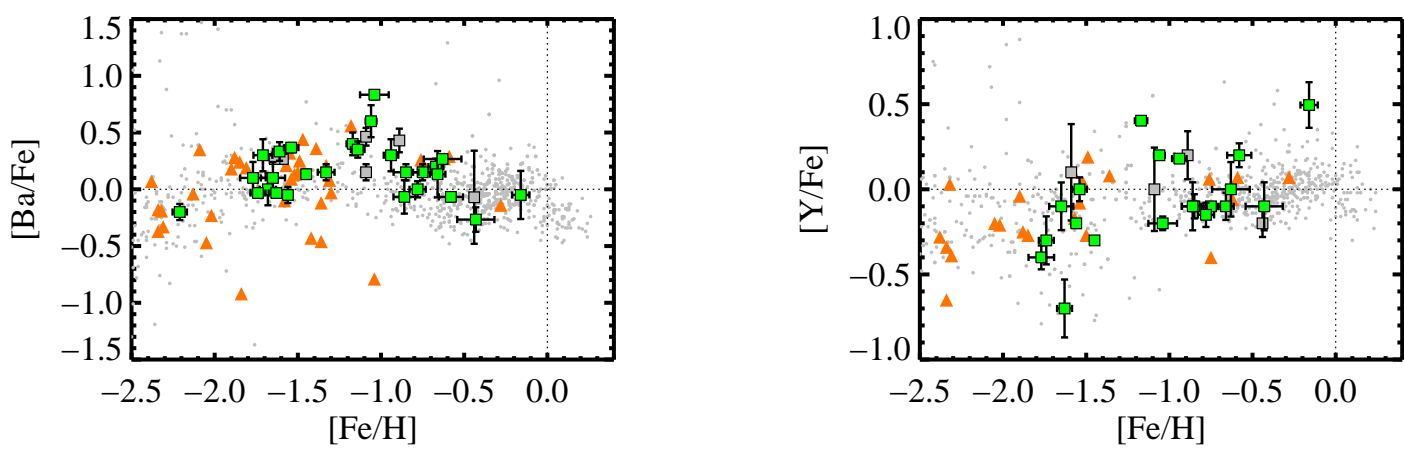

Figure 3: $[\mathrm{Ba} / \mathrm{Fe}]$ and $[\mathrm{Y} / \mathrm{Fe}]$, symbols are the same as in Figure 2.
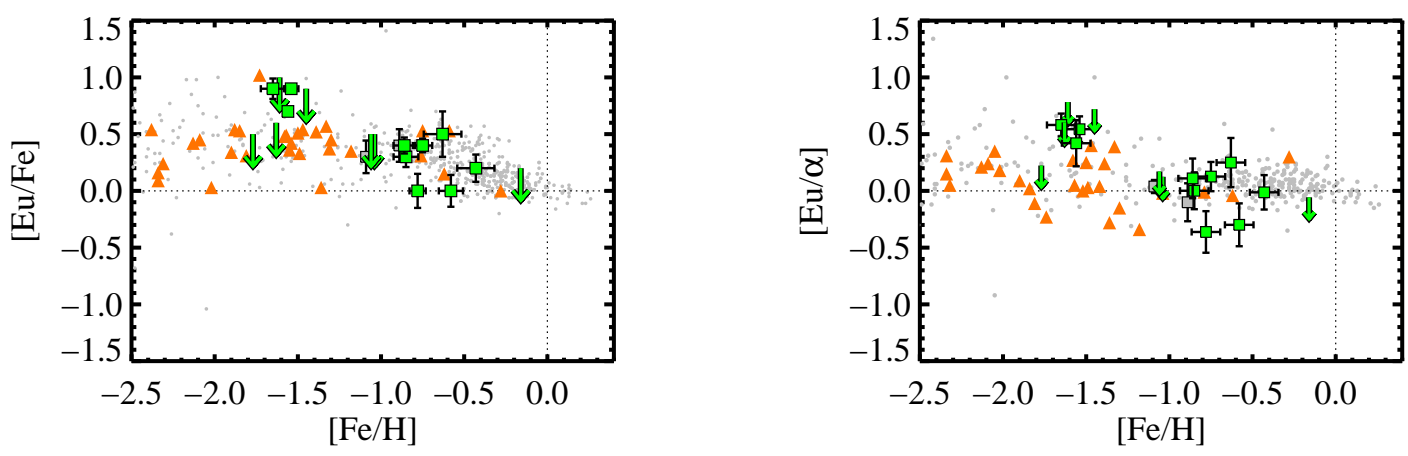

Figure 4: $[\mathrm{Eu} / \mathrm{Fe}]$ and $[\mathrm{Eu} / \alpha]$, symbols are the same as in Figure 2.

distribution for GCs outside $30 \mathrm{kpc}$, with a mean metallicity of $[\mathrm{Fe} / \mathrm{H}]=-1.6$, which was estimated from line indexes. We also note that at least one GC in our sample has an intermediate age (1-3 Gyr) at high $[\mathrm{Fe} / \mathrm{H}]$; this cluster has disk-like kinematics [15] and lies within $7 \mathrm{kpc}$.

In Figures 2-4, we show the $[\mathrm{X} / \mathrm{Fe}]$ as a function of $[\mathrm{Fe} / \mathrm{H}]$ for $\mathrm{Ca}, \mathrm{Si}, \mathrm{Ba}, \mathrm{Y}$, and $\mathrm{Eu}$. For the $\alpha$-elements $\mathrm{Ca}$ and $\mathrm{Si}$, we find a plateau value that is similar to the value in MW GCs and field stars from [16, 17], which is consistent with rapid early star formation in M31. Note that our results do not confirm the line index results from $[18,19]$. The underestimations of $[\alpha / \mathrm{Fe}]$ in $[18,19]$ are likely due to $\mathrm{Mg}$ depletion within some of the cluster stars; we first published evidence of this effect being visible in the integrated light of GCs in $[4,7]$. The star-to-star variations in the light elements $\mathrm{Na}, \mathrm{O}, \mathrm{Mg}$, and $\mathrm{Al}$, are a well known phenomenon, and discussed in the review by 

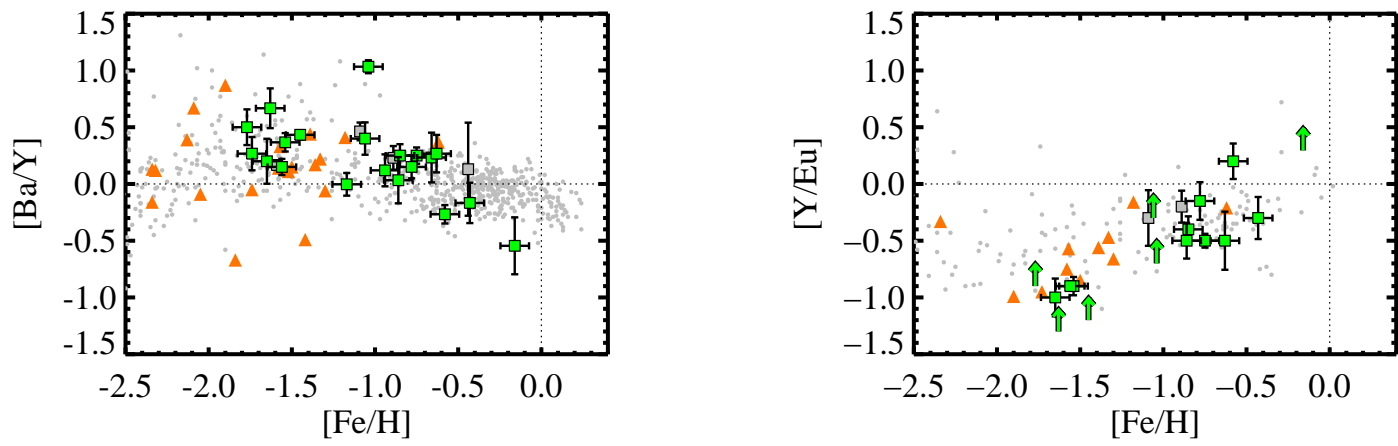

Figure 5: $[\mathrm{Ba} / \mathrm{Y}]$ and $[\mathrm{Y} / \mathrm{Eu}]$, symbols are the same as in Figure 2.

[20]. Most recently [21] confirmed, using a slightly different IL analysis, that star-to-star variations in $\mathrm{Mg}$ are detectable in IL, and therefore $\mathrm{Mg}$ is not a good proxy for $[\alpha / \mathrm{Fe}]$ in IL GC spectra. In Figure 2 there is a clear knee visible in the $[\mathrm{Ca} / \mathrm{Fe}]$ values, which closely matches the abundance patterns of MW field stars. Obvious outliers in the abundances of our GC sample include at least one $\mathrm{GC}$ with high $[\mathrm{Fe} / \mathrm{H}]$ and high $[\alpha / \mathrm{Fe}]$, and one $\mathrm{GC}$ with low $[\mathrm{Fe} / \mathrm{H}]$ and low $[\alpha / \mathrm{Fe}]$, the latter of which may be associated with an accretion event [22].

Note that the abundances shown in Figures 3 and 4 are the first results for $r$ - and s-process abundances in old populations in M31. In general, we find that the $\mathrm{Ba}, \mathrm{Y}$, and Eu are consistent with MW heavy element abundances. The $[\mathrm{Eu} / \mathrm{Fe}]$ values for $\mathrm{GCs}$ with $[\mathrm{Fe} / \mathrm{H}]<-1.5$ show some suggestion of being higher than the mean of MW GCs, although they are still within the range exhibited by the MW. A larger sample of low metallicity Eu abundances is necessary to confirm this result.

In Figure 4 - 5 we show key neutron capture abundance ratios as a function of overall metallicity. These are particularly important diagnostics as $[\mathrm{Ba} / \mathrm{Y}]$ identifies the yield of second peak to first peak s-process elements, while [Y/Eu] identifies the yield of light s-process to r-process elements. $[\mathrm{Eu} / \alpha]$ is a diagnostic for different elements that are primarily produced in Type II supernovae. We find that this sample of M31 GCs is dominated by r-process enrichment at low metallicity, and the s-process influence appears to increase around $[\mathrm{Fe} / \mathrm{H}] \sim-1$, similar to the $\mathrm{MW}$. These results, like the $[\alpha / \mathrm{Fe}]$ results, are consistent with rapid, early star formation in M31.

In summary, we present ages and abundances of $\mathrm{Fe}, \mathrm{Ca}, \mathrm{Si}, \mathrm{Ba}, \mathrm{Y}$, and $\mathrm{Eu}$ for a total sample of 27 GCs in M31. For the abundances shown here, M31 generally looks like the MW; even this basic similarity has been controversial to assert from previous line index analyses. Our analysis of the IL spectra of extragalactic GCs demonstrates the importance of quantitative, accurate abundance ratios in clarifying the chemical enrichment and formation history of galaxies beyond the Milky Way. M31 is the first of many galaxies within the Local Group and nearby Groups that we are studying with this technique.

\section{References}

[1] Searle, L., \& Zinn, R., Compositions of halo clusters and the formation of the galactic halo, ApJ, 225, 357 (1978)

[2] Bernstein, R. A., \& McWilliam, A., A New Method for Measuring the Detailed Chemical Composition of Globular Clusters from High-Resolution, Integrated-Light Spectra, Extragalactic Star Clusters, 207, 739 (2002) 
[3] McWilliam, A., \& Bernstein, R. A., Globular Cluster Abundances from High-Resolution Integrated-Light Spectra. I. 47 Tuc, ApJ, 684, 326 (2008)

[4] Colucci, J. E., Bernstein, R. A., Cameron, S., McWilliam, A. \& Cohen, J. G., M31 Globular Cluster Abundances from High-Resolution, Integrated-Light Spectroscopy, ApJ, 704, 385 (2009)

[5] Cameron, S. A., The development of a detailed abundance analysis method intended for the integrated light spectra of extragalactic globular clusters, PhD thesis, University of Michigan (2009)

[6] Colucci, J. E., Bernstein, R. A., Cameron, S. A., \& McWilliam, A., Globular Cluster Abundances from High-resolution, Integrated-light Spectroscopy. III. The Large Magellanic Cloud: Fe and Ages, ApJ, 735, 55 (2011)

[7] Colucci, J. E., Bernstein, R. A., Cameron, S. A., \& McWilliam, A., Globular Cluster Abundances from High-resolution, Integrated-light Spectroscopy. IV. The Large Magellanic Cloud: $\alpha, F e-p e a k$, Light, and Heavy Elements, ApJ, 746, 29 (2012)

[8] McWilliam, A., Preston, G. W., Sneden, C., \& Shectman, S., A Spectroscopic Analysis of 33 of the Most Metal-Poor Stars.I., AJ, 109, 2736 (1995)

[9] Pietrinferni, A., Cassisi, S., Salaris, M., \& Castelli, F., A Large Stellar Evolution Database for Population Synthesis Studies. II. Stellar Models and Isochrones for an $\alpha$-enhanced Metal Distribution, ApJ, 642, 797 (2006)

[10] Sneden, C., The nitrogen abundance of the very metal-poor star HD 122563 ApJ, 184, 839 (1973)

[11] Galleti S., Federici L., Bellazzini M., Fusi Pecci F., Macrina S., 2MASS NIR photometry for 693 candidate globular clusters in M31 and the Revised Bologna Catalogue, A\&A, 416, 917 (2004)

[12] Caldwell, N., Schiavon, R., Morrison, H., Rose, J.A., \& Harding, P., Star Clusters in M31. II. Old Cluster Metallicities and Ages from Hectospec Data, AJ, 141, 61 (2011)

[13] Alves-Brito, A., Forbes, D. A., Mendel, J. T., Hau, G. K. T., \& Murphy, M. T., The outer halo globular clusters of M31, MNRAS, 395, L34 (2009)

[14] Huxor, A. P., et al., Exploring the properties of the M31 halo globular cluster system, MNRAS, 414, $770(2011)$

[15] Morrison, H. L., Harding, P., Perrett, K., \& Hurley-Keller, D., M31's Undisturbed Thin Disk of Globular Clusters, ApJ, 603, 87 (2004)

[16] Venn, K.A., Irwin, M., Shetrone, M.D., Tout, C.A., Hill, V., \& Tolstoy, E., Stellar Chemical Signatures and Hierarchical Galaxy Formation, AJ, 124, 1177 (2004)

[17] Pritzl, B. J., Venn, K. A., \& Irwin, M., A Comparison of Elemental Abundance Ratios in Globular Clusters, Field Stars, and Dwarf Spheroidal Galaxies , AJ, 130, 2140 (2005)

[18] Beasley, M. A., Brodie, J. P., Strader, J., et al., The Chemical Properties of Milky Way and M31 Globular Clusters. I. A Comparative Study, AJ, 128, 1623 (2004)

[19] Puzia, T. H., Perrett, K. M., \& Bridges, T. J., New light on the formation and evolution of M 31 and its globular cluster system, A\&A, 434909 (2005)

[20] Gratton, R., Sneden, C., \& Carretta, E., Abundance Variations Within Globular Clusters, ARAA, 42, 385 (2004)

[21] Larsen, S. S., Brodie, J. P., \& Strader, J., Detailed abundance analysis from integrated high-dispersion spectroscopy: Globular clusters in the Fornax Dwarf Spheroidal, eprint arXiv:1209.1092, (2012)

[22] Mackey, A. D., Huxor, A. P., Ferguson, A. M. N., et al., Evidence for an Accretion Origin for the Outer Halo Globular Cluster System of M31, ApJL, 717, L11 (2010) 